\title{
Nachhaltige Medizin - Facette einer nachhaltigen Gesellschaft
}

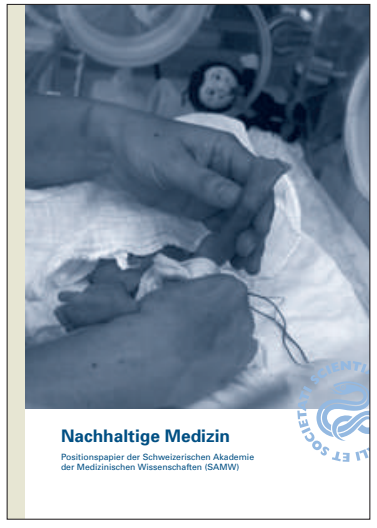

* Anmerkung zur öffentlichen Gesundheit: Als kurz nach der Wiederwahl von Barack Obama dieser Artikel entstand, verkündete ein republikanischer Kongressleader, dass seine Partei den dummen, aufreibenden Widerstand gegen das neue Gesundheitsgesetz aufgeben würde und versprach für die Zukunft: «Obamacare is the law.» Eine gute Nachricht; denn es geht um ein Gesetz, das die Zahl der Amerikaner ohne Krankenversicherung um 30 Millionen senkt.

1 SAMW. Nachhaltige Medizin. Schweiz Ärztezeitung. 2012;93(45):1645-53.

2 Taverna E. Mehrwert «Spiritualität». Schweiz Ärztezeitung. 2012;93(45): 678 .
Wir sollten der Schweizerischen Akademie der Medizinischen Wissenschaften SAMW dankbar dafür sein, dass sie einen Bericht über die Notwendigkeit einer nachhaltigen Medizin initiiert hat [1]. Allerdings wird er nicht auf jedermanns Zustimmung stossen; denn wenn man konstatiert, «der Nutzen medizinischer Interventionen ist nicht immer vorhanden, oft wird er überschätzt», «das Gesundheitswesen setzt oft falsche Anreize», oder wenn man «anachronistische standespolitische Besitzstände» kritisiert, wird man der Selbstgeisselung und des Defaitismus bezichtigt.

Anzuerkennen ist, dass der Bericht unser Gesundheitssystem aus einem anderen Blickwinkel betrachtet und unseren Beruf als Mischung aus Handwerk, Wissenschaft, Kunst und Profit, wie E. Taverna es sagt [2], in den gesamtgesellschaftlichen Kontext stellt.* Wichtig ist, dass eine angesehene Instanz Position bezieht und zu einem Whistleblower wird.

Denn so kompetent und klug Ärzte auch sind, so können sie die notwendigen Veränderungen selbstverständlich nicht im Alleingang herbeiführen. Aktive Einbeziehung des Patienten ist heute in aller Munde. Angesichts der angestrebten Entwicklung und zahlreicher daran beteiligter Stakeholders hat jeder seine Aufgabe zu übernehmen: In der Kooperation zur Optimierung des Therapieerfolgs (um die Zahl nutzloser Leistungen zu reduzieren, dürfen sie nicht erst angefordert werden!) und auch durch Beteiligung an der politischen Debatte zur Schaffung eines Gesundheitswesens ohne kontraproduktive Anreize und zur Förderung einer vernünftigen Gesundheitsplanung. Das setzt Erziehung zu staatsbürgerlicher Gesinnung und massvoller Nutzung des Gesundheitssystems voraus und dies bereits in der Schule. Es bedeutet auch, dass man sich der mittel- und langfristigen Konsequenzen, z.B. der später eintretenden Nebenwirkungen, bewusst wird.

Politiker sollten aus einem zukunftstorientierten Blickwinkel auf der Grundlage der Qualität ihrer Arbeit und ihrer Vision beurteilt werden, statt sie zum Rücktritt $\mathrm{zu}$ bewegen, weil sie eine schwierige $\mathrm{Zu}$ kunft voraussagen. Politikern würde eine solche Einstellung ermöglichen, von übertriebenen Wahlversprechen abzuweichen. Denn im Allgemeinen ist man weit davon entfernt (meine Zeitung titelte zur Wahlkampagne in den USA: «Geld und Lügen verminen die amerikanische Demokratie». Well ...).

Und man sollte einen gewissen Abstand gewinnen, z. B. gegenüber den Wundern, die das «Human Enhancement» verspricht. Dies soll nicht heissen, dass man Massnahmen zur Förderung der Gesundheit verwirft, sofern man nicht - wie es zwischen den Zeilen allzu häufig anklingt - dem Traum der Unsterblichkeit verfällt (m. E. ein Alptraum). Spricht man von einer nachhaltigen Medizin, so muss man die Gefahr der Diskriminierung abschätzen: Denn es werden die «best, brightest and richest» sein, die davon profitieren, und es wird schwierig sein, die Kluft zu den «Otto Normalpatienten» zu schliessen. Überdies (horribile dictu) muss eine nachhaltige Medizin, die zum Ziel hat, «dass auch spätere Generationen von guten medizinischen Leistungen profitieren können» (so im ersten Absatz des SAMW-Positionspapiers), einige mit dem Begriff Enhancement verbundene Sichtweisen mit kritischem Blick betrachten. Besonders weil die späteren Generationen (die wohlgemerkt kein Wahlrecht haben) in dieser Welt nicht willkommen geheissen werden. Denn sie verstärken den Druck auf die Lebenswelt, und die ältere Generation, die dann so lange so gut leben wird, wird dies kaum begrüssen ... Der Akademie zufolge fehlt zunehmend die Einsicht in die «Unaufhebbarkeit der «condition humaine` als Einbettung des Lebens zwischen Geburt und Tod». In klaren Worten: Die angestrebte Nachhaltigkeit setzt Ablösung und Erneuerung voraus, nicht Zementierung des Bestehenden und dies gilt auch für die Menschheit! Ich weiss nicht mehr, von wem der Satz stammt: «Im Wissen um unsere Sterblichkeit gründet unsere Freiheit.»

Nachhaltigkeit hat eine Vernunftsdimension, jedenfalls bedeutet sie nicht übereilte Flucht nach vorne. Nachhaltige Medizin kann nur eine (wichtige) Facette einer insgesamt nachhaltigen Gesellschaft sein. Um dies zu erreichen, muss man sich vom Mantra des Wachstums verabschieden. Und etwas Zurückhaltung üben. Obama II lässt hoffen, dass sich die USA künftig erkennbarer gegen die Klimaerwärmung engagieren werden. Doch werden wir selbst schnell und kräftig genug die Bremse ziehen können?

Im Positionspapier sind viele sinnvolle Massnahmen aufgeführt. Aber andererseits ist allgemein bekannt, dass - sei es in der Arztpraxis oder im Leben der res publica - die Menschen bei unangenehmen Nachrichten nicht richtig hinhören. Und die Worte der Akademie werden von all jenen als unangenehm empfunden, die sich mit dem Gedanken der Begrenztheit und Genügsamkeit nicht anfreunden können und die nur sehen wollen, wie die Medizin triumphiert. Danken wir jenen, die ihren Zauberstab heben, um uns mit dem Willen zu Nachhaltigkeit zu behexen - damit wir die «innovativen Lösungen» finden, wie sie das Positionspapier fordert.

Jean Martin, Mitglied der Nationalen Ethikkommission und der Redaktion 